\title{
DIABÉTICOS MEXICANOS Y EL SISTEMA DE SALUD PÚBLICA: RECUENTO DE LOS DAÑOS - 2016
}

\author{
DIABÉTICOS MEXICANOS E O SISTEMA DE SAÚDE PÚBLICA: CONTAGEM \\ DOS DANOS - 2016
}

\section{MEXICAN DIABETICS AND THE PUBLIC HEALTH SYSTEM: COUNTING DAMAGES - 2016}

\author{
Tomás Serrano Avilés*
}

\begin{abstract}
RESUMEN
En México, la transición epidemiológica se caracteriza por el inusitado aumento de la diabetes y la obesidad. Ambas enfermedades están asociadas y representan la oportunidad de intervenir con política pública. Los servicios de sanidad explican una pequeña parte de las diferencias en salud entre una población y otra. El objetivo general del trabajo es reflexionar sobre la importancia de la diabetes como posibilidad de análisis de la política pública en salud en México. Las encuestas nacionales (2012 y 2016) se usan como referencia empírica para mostrar que la amputación se concentra en los trabajadores informales o desempleados. El trabajo es sugerente al observar el mal desempeño de los servicios de sanidad, que requieren resolver el problema público aquí identificado.
\end{abstract}

Palabras-clave: Derechos humanos, políticas públicas, salud, diabetes, obesidad

\section{RESUMO}

Em México, a transição epidemiológica caracteriza-se pelo inusitado aumento da diabetes e da obesidade. Ambas as doenças estão associadas e representam uma oportunidade de intervir através de políticas públicas. Os serviços de atenção médica explicam uma pequena parte das diferenças em saúde entre uma população e outra. O objetivo geral do trabalho é reflexionar sobre a importância da diabetes como possibilidade de análise da política pública em saúde em México. As pesquisas nacionais (2012 e 2016) são usadas como referência empírica para mostrar que a amputação se concentra nos trabalhadores informais ou desempregados. O trabalho sugere observar o mau desempenho dos serviços de previdência, que requerem resolver o problema público aqui identificado.

Palavras-chave: Direitos humanos, políticas públicas, saúde, diabetes, obesidade

\begin{abstract}
In Mexico, the epidemiological transition is characterized by the unusual increase in diabetes and obesity. Both diseases are associated and represent the opportunity to intervene with public policy. Health services explain a small part of the health differences between one population and the other. The general objective of this work is to reflect on the importance of diabetes as a possibility of analysis of the public health policy in Mexico. National surveys (2012 and 2016) are used as
\end{abstract}

“Profesor de la Universidad Autónoma del Estado de Hidalgo (México).Doctor en ciencias sociales por el Colegio de la Frontera Norte. 
an empirical reference to show that amputation is concentrated in informal or unemployed workers. This work is suggestive in observing the poor performance of health services, which require solving this public problem identified here.

Keywords: Diabetes, obesity, health, public policies, human rights.

\section{INTRODUCCIÓN}

Las reflexiones que transitan en el tema de los derechos humanos son sumamente complejas. Su estudio puede apreciarse mejor desde la perspectiva de las políticas públicas, pues el asunto tiene claras implicaciones prácticas en diversos campos de estudio. En este trabajo el objetivo general es reflexionar sobre la importancia de la diabetes como posibilidad de análisis de la política pública de salud en México (2012-2016). Los objetivos particulares son describir la frecuencia de la diabetes y la obesidad, e, identificar evidencia de la falta de respeto a los derechos humanos de los diabéticos.

Por un lado, vale la pena señalar que el trabajo de ningún modo aspira dar cuenta del estado de salud de los mexicanos, ni tampoco de la situación de los servicios de sanidad del país. Dada la estrecha relación entre la diabetes y la alimentación se constata este comportamiento para sugerir nuevas estrategias de atención del proceso salud-enfermedad.

La mayoría de los países de América Latina se encuentran en una etapa de transición epidemiológica, la cual se caracteriza por el cambio de enfermedades transmisibles a las crónicas no transmisibles, fuentes principales de morbilidad y mortalidad. Estos países además comparten a su vez el cambio demográfico (FRENK, LOZANO y BOBADILLA, 1994).

En países desarrollados y en desarrollo hay una tendencia reciente que sostiene hay mecanismos multicausales de la enfermedad, tales como la genética, transtornos del sueño, estrés, sedentarismo, etc. Sin embargo, existe un amplio consenso que la alimentación es la clave de la obesidad, y ésta a su vez, es factor determinante de las enfermedades crónicas de mayor mortalidad, entre las que destacan las enfermedades cardiovasculares y la diabetes, aspecto en el que es posible intervenir (FRENK, 1995).

En México, de acuerdo al instituto nacional de geografía e informática, en el 2016, las 10 principales causa de muerte de los mayores de 20 años ocurrieron en el siguiente orden de importancia: enfermedades del corazón, diabetes, tumores malignos, enfermedades del hígado, accidentes, enfermedades cerebrovasculares, agresiones, enfermedades pulmonares, influenza y neumonía, e insuficiencia renal.

En el país, la diabetes ha sido considerada como una epidemia (HERNÁNDEZ, GUTIÉRREZ y REYNOSO, 2013; SHAW, 2006; BOSCH, ALFONSO y BERMEJO, 2002). De acuerdo a Hernández, Gutiérrez y Reynoso (2013), en el 2012, cerca del 10\% de los adultos ha recibido un diagnóstico de diabetes. Los datos son contrastantes. El mínimo es del 5.6\% en Chiapas y el valor máximo es del $12.3 \%$ en el Distrito Federal. Del total de diabéticos, $46.95 \%$ reportó a su vez diagnóstico de hipertensión, $4.47 \%$ antecedente de infarto del corazón y el $54.46 \%$ tenía antecedentes familiares de diabetes.

Según la secretaría de salud (2016), la diabetes tiende a crecer del 2012 al 2016. Esta misma fuente sostiene que debido a la mala calidad y alta demanda en el servicio médico público ha incrementado el uso de los servicios privados, aún a pesar de que la población se encuentra afiliada a alguna de las instituciones públicas. En este año, la tasa de hospitalizaciones por diabetes mellitus fue de 329 por cada 100,000 habitantes y 96 por hipertensión arterial, valores por encima del promedio de la Organización para la Cooperación y el Desarrollo Económico (OCDE), (150 y 83, respectivamente). A su vez, en 2016, el número de médicos per cápita fue de 1 por cada 1,000 habitantes. El sector privado concentró el $69 \%$ del total de quirófanos y el público sólo el $31 \%$. Los quirófanos son salas en unidades de hospitalización que cuentan con equipo médico adecuado para llevar a cabo cirugías.

En primer lugar el trabajo es un reto porque observa la parte de la población que carece en términos reales de acceso a los servicios básicos de salud.

La investigación es sugerente además porque la salud, en tanto un derecho humano habrá de ser universal. El artículo 25 de la declaración universal de 
los derechos humanos de las naciones unidas (1948) la establece por primera vez como ideal fundamental:

Toda persona tiene derecho a un nivel de vida adecuado que le asegure, así como a su familia, la salud y el bienestar, y en especial la alimentación, el vestido, la vivienda, la asistencia médica y los servicios sociales necesarios; tiene asimismo derecho a los seguros en caso de desempleo, enfermedad, invalidez, viudez, vejez y otros casos de pérdida de sus medios de subsistencia por circunstancias independientes de su voluntad.

En tercer lugar es importante conocer la realidad por la diversidad de cobertura. Por lo que las instituciones de salud deben buscar distintas formas de mejorar la evaluación y participación de la población, en especial la de los trabajadores, pues los resultados obtenidos identifican este sector con el problema prioritario, dadas las condiciones sanitarias. Son ellos los que se tienen que organizar para tener mejores condiciones en sus centros de trabajo, tales como el descanso, la activación y la alimentación.

En este sentido, el artículo 24 de la declaración de los derechos humanos señala:

Toda persona tiene derecho al descanso, al disfrute del tiempo libre, a una limitación razonable de la duración del trabajo y a vacaciones periódicas pagadas.

Los resultados empíricos de la atención a la salud (mayores de 20 años) señalan la ocurrencia mayoritariamente a la edad laboral y la incidencia de las amputaciones se concentra en la población con empleo informal o desempleada, en referencia a la parte que tiene empleo formal y con cobertura médica segura.

Por lo tanto, el artículo se estructura en el siguiente contenido: el contexto sanitario, antecedentes de la diabetes y la obesidad, la política pública, materiales y métodos, el problema sanitario de los diabéticos, discusión y reflexiones finales.

\section{EL CONTEXTO SANITARIO}

La teoría de la transición epidemiológica parte de la teoría de la transición demográfica para explicar los cambios en los patrones de salud y enfermedad que se originan en determinadas condiciones demográficas, económicas y sociales (LADROVE, 2005).

La transición epidemiológica se caracteriza por pasar de patrones de morbimortalidad por causas agudas - principalmente infecciosas-a la emergencia de enfermedades crónicas no transmisibles, también llamadas silenciosas, entre las que destacan la diabetes y el cáncer. La complejidad en las causas puede sintetizarse en el problema de la obesidad, y ésta a su vez está determinada por la alimentación. Al respecto, se propone intervenir en edades tempranas, siempre que se pretenda mitigar el impacto que la situación actual tendrá en el futuro (DURÁN, 2005).

Es así como en México, en la transición epidemiológica predominan las enfermedades no transmisibles. Los padecimientos emergentes que constituyen las principales causas de muerte: diabetes, enfermedades isquémicas del corazón, padecimientos cerebrovasculares y cáncer. En 2008, la principal causa de defunción en hombres y mujeres en todo el país fue la diabetes y su prevalencia en adultos fue del 14.4\%, una de las más altas del mundo (GÓMEZ, et al., 2011).

El aumento de la esperanza de vida y la creciente exposición a los llamados riesgos emergentes relacionados con estilos de vida poco saludables han modificado el cuadro de las principales causas de muerte (GÓMEZ, et al., 2011).

En relación a la deficiente cobertura y mala calidad en el servicio de salud es posible, sea la causa que explica la ocurrencia tal elevada de las defunciones en México. A diferencia, en el mismo año, en los Estados Unidos, de acuerdo a Heron (2018), la diabetes fue la octava causa de muerte, lo cual se debe a que la población tiene garantizada la cobertura en los servicios de salud en aquel país.

La secretaría de salud (2016) reconoce que el Sistema Nacional de Salud no ha sido capaz de proporcionar servicios sanitarios a la población que no cuenta con un empleo formal, ni tampoco ha logrado mejorar su calidad.

El sistema de salud se compone de instituciones públicas y privadas. Las primeras se financian con contribuciones gubernamentales, por el empleador (Imss, Issste, Pemex, Sedena y Semar) y por los propios empleados. Adicionalmente, la población tiene cobertura a través del seguro popular que se financia por una cuota social que cubre el gobierno federal y estatal. El sector privado obtiene su financiamiento de los pagos de bolsillo de las personas al momento de recibir la atención y al adquirir medicamentos e insumos (GÓMEZ, et al., 2011). 
Los datos oficiales de 2016 (SECRETARIA DE SALUD, 2016) indican que la afiliación de la población total a alguna institución de salud fue sólo del 13.4\%. Es destacable además que el diagnóstico oficial, describe un mundo perfecto por donde se le mire. Por ejemplo, las condiciones de los establecimientos de salud fueron evaluados por los pacientes con buenas o muy buenas $(67.9 \%)$ o regulares condiciones $(27.2 \%)$. O que el $87.1 \%$ de ellos recibió prescripción médica, y que -de éstos- el $77.1 \%$ recibió los medicamentos gratuitos en su totalidad.

\section{ANTECEDENTES DE LA DIABETES Y LA OBESIDAD}

Entre las variables explicativas más importantes de las investigaciones que analizan la diabetes destacan las que asocian la enfermedad con la obesidad. Según los estudios realizados, el estado de salud-enfermedad se ha resuelto de manera interdisciplinaria.

Las investigaciones destacan el papel de la alimentación como mecanismo causal y de intervención de las enfermedades crónicas, incluyendo el cáncer. De acuerdo a Julio Frenk (1991), la diabetes se tiene que pensar desde una perspectiva integral, partiendo del conocimiento del conjunto de factores demográficos, epidemiológicos, económicos, sociales, políticos que la determinan. El mayor problema es que al igual que otros modelos sigue siendo difícil desagregar la interacción que ocurre entre las variables.

A nivel mundial, hay evidencia de que la obesidad aumenta los riesgos de diabetes y de algunos tipos de cáncer. En este caso, se tienen resultados contrastantes acerca de algunos medicamentos que se usan en el tratamiento de la diabetes que aumentan, mientras otros disminuyen el riesgo al cáncer (KLIL, et al., 2016).

En Cuba, la emergencia de las enfermedades crónicas no transmisibles son el problema prioritario porque es la principal causa de muerte de la población envejecida (LANDROVE, 2005).

Para Mondini et al. (2007), en su estudio realizado con niños de primer grado en la región de Cajamar (Sao Paulo, Brasil), en 2005, se encontró asociación entre sobrepeso y las variables socioambientales. El 17\% de los niños fueron definidos con sobrepeso. Los factores causales fueron: obesidad materna, alto consumo de comida procesada, pasar más de 4 horas al día viendo televisión y el consumo de grasas en el hogar. El estudio sugiere que el mecanismo de la obesidad en Brasil es por el cambio en la alimentación, ya que disminuyó la ingesta de arroz y frijol e incrementó el consumo de productos procesados, tales como galletas, helados y refrescos.

En Argentina, en el 2009, el 53,4\% de los adultos entrevistados presentó exceso de peso (sobrepeso y obesidad). En esta oportunidad se observó mayor prevalencia de obesidad en las edades de 50 y 64 años, en lo de menor ingreso y menor nivel educativo. El nivel de diabetes fue del $9.6 \%$ y el 34.5\% tenían presión alta. Las enfermedades crónicas no transmisibles se encontraron relacionadas con aproximadamente el $80 \%$ de la mortalidad general. Los datos usados fueron de la encuesta nacional de factores para la vigilancia de enfermedades no transmisibles (FERRANTE, et al., 2011).

Con base en datos recopilados por el Instituto Nacional de Estadística de Guatemala (2009), en el municipio de Villa Nueva se entrevistaron a 1,545 personas seleccionadas por muestreo en etapas múltiples y estratificadas en dos grupos de edades ( 20 a 39 años y 40 años o más). El porcentaje de mortalidad por enfermedades crónicas no transmisibles fue de casi el $60 \%$, se incluye el $38 \%$ de muertes por enfermedades cardiovasculares y $22 \%$ por diabetes. Asimismo, la prevalencia total de diabéticos fue de 8.4\%. El 4.3\% de los encuestados presentaron diabetes conocida, mientras que $4.1 \%$ fueron diagnosticados como nuevos casos.

En España se observó la tendencia al aumento del sobrepeso y la obesidad en la población adulta, entre 1987 y 2012, especialmente entre los hombres. En general, en el periodo de estudio, el sobrepeso pasó del 34.0 a $35.8 \%$ y la obesidad se duplicó $(8.0 \%$ a 16.5\%). Para este fin, se usaron datos de 8 encuestas nacionales de salud, del tipo transversal (BASTERRA, et al., 2017).

En los Estados Unidos se han encontrado resultados alentadores que indican que disminuyendo paulatinamente la obesidad se retrasar la progresión de la prediabetes a la diabetes tipo 2 y también mejora la salud para quien tiene diagnosticada la diabetes tipo 2. El mecanismo consiste en la pérdida de peso moderada, que mejora el control glucémico y reduce la necesidad de medicamentos hipoglucemiantes. Mediante esta intervención, se 
sugieren recomendaciones dietéticas, farmacológicas y quirúrgicas con que disminuye la obesidad, y con ésta, mejora el control de la diabetes tipo 2 (AMERICAN DIABETES ASOCIATION, 2017).

En México, con base en el análisis de la información publicada de 1980 a 2013, la prevalencia de la obesidad se estimó triplicada, principalmente entre las mujeres, dado el patrón característico de alimentación. Esta enfermedad se sugiere, incrementa la prevalencia a la hipertensión arterial, a la diabetes y al cáncer (REYNOSO, et al., 2018).

En este panorama, Estébanez (2002) sugiere que en el mundo hay una crisis general en la salud de la población. Lo mismo ocurre en los países desarrollados como en los de desarrollo. La mejor alternativa es recurrir a la medicina humanitaria para intervenir en situaciones de violación de los derechos humanos y apoyar a la asistencia de las víctimas. La característica principal es que hay una enorme desigualdad de acceso a los servicios sanitarios. Al respecto se citan casos brutales que ejemplifican la negación de los derechos básicos a la salud, con base en que imperan prejuicios y diferencias de género, clase y edad.

\section{LA POLÍTICA PÚBLICA}

Las políticas públicas parten del supuesto que el Estado tiene que intervenir para resolver las fallas en el mercado, $\mathrm{y}$, fundamentalmente para solucionar los problemas públicos emergentes. Entonces, sólo la participación del Estado hace posible la existencia de las políticas públicas. De modo que si el objetivo es resolver los problemas públicos, todo lo que haga o deje de hacer el Estado es política pública (MERINO, 2013).

Según Franco y Álvarez (2008), promover el respeto a los derechos humanos por medio de una mayor comprensión de éstos, refuerza la importancia de las políticas públicas. La investigación en políticas de salud ha mantenido como objeto de estudio la salud poblacional y los sistemas de salud como centro de intervención. Los derechos humanos son el marco apropiado para la acción en salud pública. Históricamente, primero aparecieron los derechos civiles (siglo XVII), luego los derechos políticos, y al final se desarrollaron los derechos sociales (siglo $\mathrm{XX}$ ). Los derechos humanos son el marco correcto para la práctica. Las vías de acción en salud púbica sirven para abatir la pobreza, la marginación, la desigualdad, las diferencias de género y la violación de los derechos humanos. Las políticas públicas tienen sentido sólo si se apoyan en los derechos humanos mediante mecanismos de construcción de ciudadanía y participación política.

Otro argumento adicional que sugiere la posibilidad de que las políticas públicas en salud se tienen que pensar en el marco de los derechos humanos es la aportación de Julio Frenk (1995). El autor afirma que la salud pública no es exclusivamente un campo de investigación, sino también un espacio de acción práctica, por lo que recomienda investigar las necesidades cambiantes de la población y las formas de organización que impiden la mejora en los servicios sanitarios.

Adicionalmente, el enfoque de los derechos humanos y la salud se ha desarrollado vigorosamente desde la perspectiva de la moral y/o la ética. El centro de interés ha sido definido a través de la bioética.

De esta manera, las malas prácticas que ocurren de manera cotidiana en este sector han ido abonando al campo de la demanda moral, conjuntando los problemas más escabrosos a los que se enfrentan la práctica sanitaria.

A principios de 1980, Daniel Callahan, propuso a la bioética como una herramienta adicional para el análisis de políticas públicas en salud. El enfoque se refiere a conjuntar los derechos y deberes de quien entrega y quien recibe cuidados de salud.

Sin embargo, en la actualidad, la salud pública necesita mejores datos que permitan orientar la dirección de intervención del Estado en el objetivo del respeto a los derechos humanos. En el mundo de la salud, las violaciones de los derechos básicos no prosperan en ningún marco legal institucional. De acuerdo a Mann (1996) las negligencias y errores médicos tienen que ser considerados como agresiones a la salud, por lo cual, la promoción y protección de los derechos humanos están relacionados con la protección y promoción de la salud también.

Recientemente, algunas teorías de la justicia distributiva contemplan la introducción de los derechos económicos y sociales como unidades de distribución. El derecho a la subsistencia indica que nadie puede disfrutar de algún derecho cuya protección exige la intervención de las instituciones si carece de lo indispensable para llevar una vida activa y saludable (DIETERLEN, 2016, p.12) 


\section{MATERIALES Y MÉTODOS}

El análisis de esta investigación usa los datos de la Encuesta Nacional de Salud y Nutrición 2012 y 2016 (ENSANUT, 2012 y 2016). Se trata de una encuesta probabilística, con un diseño de muestra polietápico y estratificado. En específico, los datos corresponden a los mayores de 20 años, que permite observar el panorama de la frecuencia, distribución de las condiciones de salud, como una estrategia para la prevención y el control del sobrepeso, la obesidad y la diabetes. Los datos corresponden a la secretaría de salud, institución que desde1986 se encarga de generar información en el objetivo de evaluar los servicios sanitarios y monitorea el aumento en la diabetes y la obesidad (SECRETARÍA DE SALUD, 2016).

Con base en esta encuesta, el primer paso fue medir la frecuencia relativa con que se identifica a los diabéticos: ¿Cuál era su edad al momento que un médico le diagnosticó la diabetes?

A continuación, para obtener la frecuencia porcentual de la obesidad se usó la pregunta ¿Qué grado de obesidad considera que tiene esta persona? En ese momento, el entrevistado eligió la respuesta con base en la ayuda de figuras corporales: ¿qué silueta corporal considera que se parece a usted en este momento? Para el caso femenino, las siluetas son claras en las opciones a, b, c, d que son mujeres delgadas, e para normales y $\mathrm{f}, \mathrm{g}, \mathrm{h}$, i con algún tipo de obesidad. Para el sexo masculino, las siluetas j, k, $1, \mathrm{~m}$ corresponden a hombres delgados, $\mathrm{n}$ normales y $\mathrm{o}, \mathrm{p}, \mathrm{q}, \mathrm{r}$ con algún tipo de obesidad. De modo que, los datos de obesidad se identificaron con base en las opciones $\mathrm{f}, \mathrm{g}, \mathrm{h}$, i para las mujeres obsesas y o, p, q, r para los hombres con obesidad.

Para la identificación del tipo de hospital se usó la pregunta ¿En qué hospital se atendió la diabetes? Las opciones de registro fueron: imss, issste, issste estatal, pemex, defensa, marina, imss prospera, hospital general, hospital civil, consultorios dependientes de farmacias, médicos privados, institutos nacionales y otros.

El cuadrol sintetiza el conjunto de variables sociodemográficas de los diabéticos usadas en este trabajo, así como la agrupación que se utilizó para medir la frecuencia relativa de los datos.

La primera variable es el sexo y se usó el registro de hombre o mujer.
Después, según la edad cumplida al diagnóstico de la enfermedad, la población se segmentó en cuatro grupos: infantes (0 a 12 años), jóvenes (13 a 29 años), adultos (30 a 64 años) y ancianos (65 y más años).

Los servicios de salud de los diabéticos se dividieron de acuerdo al derecho del trabajador en dos grandes grupos. Primero, los diabéticos terrenales son los que tienen acceso a los hospitales del imss, issste, issste estatal, pemex, defensa y marina, porque pagan cuotas salariales y tienen empleo formal. Segundo, los diabéticos del inframundo fueron definidos por el acceso a hospitales como ssa, imss prospera, hospital general, hospital civil, consultorios dependientes de farmacias, médicos privados, institutos nacionales y otros. Los últimos no pagan cuotas porque son desempleados o tienen empleo informal. La división corresponde específicamente al derecho a la salud, según el tipo de hospital al que se tiene derecho.

Cuadro 1: Listado de variables del registro de diabéticos (2012, 2016).

\begin{tabular}{l|l|}
\hline VARIABLE & $\begin{array}{l}\text { SELECCIÓN DE VARIABLES } \\
\text { Y CLAVES. }\end{array}$ \\
\hline Sexo & $\begin{array}{l}\text { Hombre (1) } \\
\text { Mujer (2) }\end{array}$ \\
\hline $\begin{array}{l}\text { Infantes (0-12 años) } \\
\text { Jóvenes (13-29 años) } \\
\text { Adultos (30-64 años ) } \\
\text { Ancianos (65 y más años ) }\end{array}$ \\
\hline $\begin{array}{l}\text { Siluetas femeninas } \\
\text { con algún grado de } \\
\text { obesidad }\end{array}$ & f, g, h, i \\
\hline $\begin{array}{l}\text { Figuras femeninas con } \\
\text { algún tipo de obesidad }\end{array}$ & o, p, q, r \\
\hline $\begin{array}{l}\text { Diabéticos terrenales } \\
\text { con derecho en } \\
\text { hospitalario como: }\end{array}$ & $\begin{array}{l}\text { imss, issste, issste estatal, pemex, } \\
\text { sedena y semar }\end{array}$ \\
\hline $\begin{array}{l}\text { Diabéticos del } \\
\text { inframundo que } \\
\text { acuden a hospitales } \\
\text { como: }\end{array}$ & $\begin{array}{l}\text { ssa, imss prospera, hospital } \\
\text { general, hospital civil, } \\
\text { consultorios dependientes de } \\
\text { farmacias, médicos privados, } \\
\text { institutos nacionales y otros }\end{array}$ \\
\hline
\end{tabular}

Vale la pena aclarar que la idea de explorar la variable amputación, como evidencia de la falta de respeto a los derechos humanos en salud viene del conocimiento empírico que indica que la atención a las heridas concentra la mayor frecuencia de ingreso de los diabéticos a los hospitales mexicanos. Con base en lo cual, decidí medir su frecuencia según dos el 
tipos de hospital al que tienen acceso las personas. El resultado fue sorprendente, pues emergió de una forma tal, como si de pronto se develara un mundo desconocido, porque no resulta fácil encontrar evidencia de la falta de respeto a los derechos humanos en salud en ninguna parte del mundo.

\section{EL PROBLEMA SANITARIO DE LOS DIABÉTICOS}

Los datos censales de 2012 y 2016 indican el aumento significativo de la diabetes de los mexicanos, pasando de $7.1 \%$ a $9.1 \%$, respectivamente. La información muestra que la enfermedad va en aumento. De acuerdo a la misma fuente, probablemente la tendencia se observa similar al comportamiento de la obesidad y las enfermedades crónicas de mayor mortalidad del país (las enfermedades cardiovasculares y el cáncer).

Respecto a los obesos, en el 2012, del total de mayores de 20 años, sólo el $6.9 \%$ de ellos tenía algún tipo de obesidad, $4.9 \%$ correspondieron a las mujeres y $2.5 \%$ a los hombres. En 2016, el nivel alcanzado fue del $41.6 \%$, distribuido con el $25.9 \%$ de las mujeres y en el $15.6 \%$ de los hombres. Entonces, hay evidencia del crecimiento acelerado de la obesidad, misma que en mayor proporción afecta al sexo femenino.

Al segmentar en dos grandes grupos el total de diabéticos de la Encuesta Nacional de Salud y Nutrición 2016 (ENSANUT, 2016), se muestra que el $60 \%$ del total son del inframundo. Éstos son los que no tenían seguro el servicio médico, a causa de no pagar cuotas salariales a los hospitales. En el orden de importancia, el 40\% de los enfermos (los terrenales) tienen cobertura sanitaria segura y son los menos (ver cuadro 2).

Gracias a la síntesis del cuadro 2, es posible apreciar a los diabéticos mexicanos según tienen cobertura médica o no. Con base en esta información, se puede notar la dimensión del problema público, ya que hay dos realidades fragmentadas, y puede haber más, dependiendo del interés de la investigación. Por un lado, es posible observar al colectivo de diabéticos socialmente más vulnerable que es el más numeroso (los del inframundo), al lado de su contraparte (los terrenales) con menor incidencia de la enfermedad.

Además, el resultado es valioso en términos de identificar la concentración de la diabetes en un grupo de edad y sexo determinados, garantía para la toma de decisiones correcta, pues, el diseño y/o modificación de la política pública tiene que hacerse para acercarse al irrestricto respeto a los derechos humanos en materia de salud.

Es destacable a su vez que cuando un médico diagnostica la diabetes a edad infantil sólo hay datos de los diabéticos terrenales y se carece de información de los del inframundo. Este resultado evidencia la deficiencia en la cobertura médica que tiene el segundo grupo, el más vulnerable. Los diabéticos del inframundo tienen una incidencia mayor en los grupos de jóvenes y adultos. Sin embargo, los diabéticos terrenales los superan en los extremos de la trayectoria de vida; es decir, hay mayor prevalencia de la enfermedad en este tipo de enfermos cuando son infantes y cuando son ancianos (Cuadro 2).

Cuadro 2: México. Edad al diagnóstico de la diabetes por un médico, 2016, según derecho de cobertura.

\begin{tabular}{|l|l|l|l|}
\hline Gpo. Edad & $\begin{array}{l}\text { \% diabéticos } \\
\text { terrenales }\end{array}$ & $\begin{array}{l}\text { \% diabéticos del } \\
\text { inframundo }\end{array}$ & \% Total \\
\hline Infantes & 100 & 0 & 100 \\
\hline Jóvenes & 48 & 52 & 100 \\
\hline Adultos & 38 & 62 & 100 \\
\hline Ancianos & 53 & 47 & 100 \\
\hline Total & 40 & 60 & 100 \\
\hline
\end{tabular}

Fuente: Cálculos propios a partir de la ENSANUD, 2016

Como se aprecia en las figuras 1 y 2, con base en la división de la población en 4 grupos de edades, la edad al diagnóstico de la diabetes se concentró en la edad laboral. Este resultado sugiere la necesidad de dirigir la política pública a este grupo poblacional, ya que, en 2016, más del 80\% fue diagnosticado con la enfermedad por un médico (los adultos). 
Figura 1: México. Pirámide de población según edad al diagnóstico de diabetes por un médico, 2012.

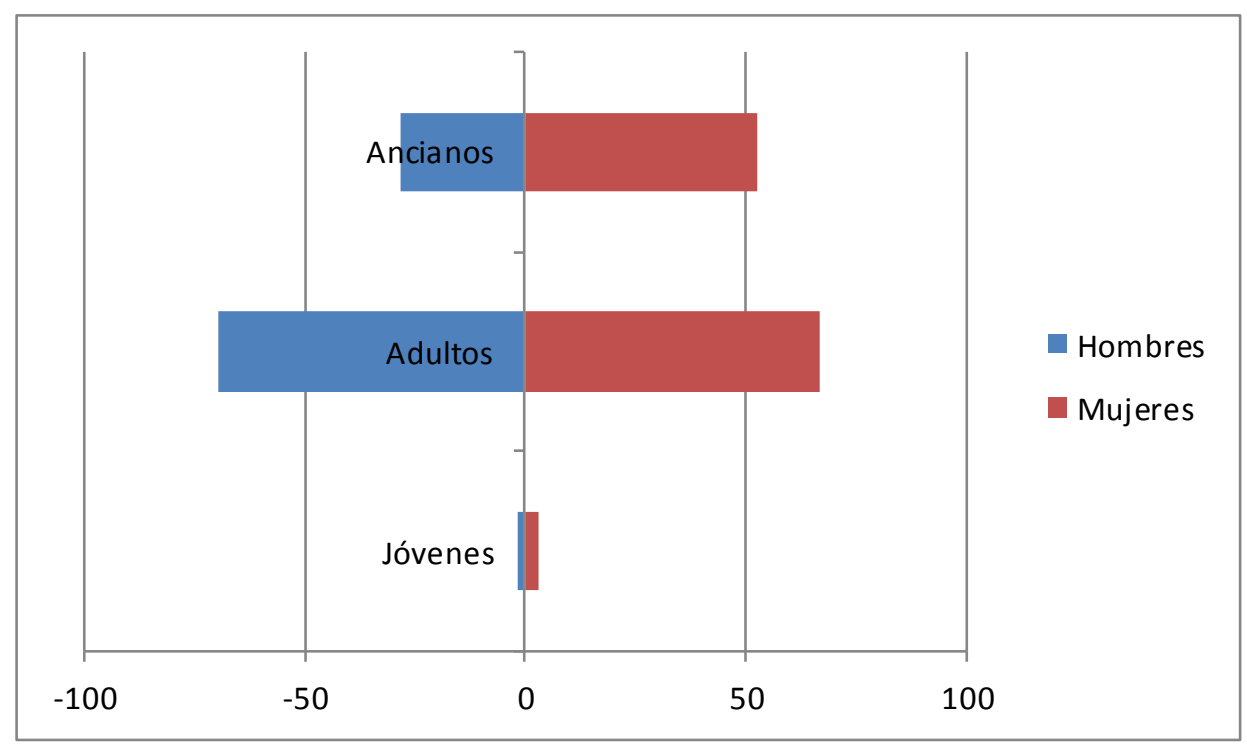

Fuente: Cálculos propios a partir de la ENSANUD, 2016.

Figura 2: México. Pirámide de población según edad al diagnóstico de diabetes por un médico, 2016.

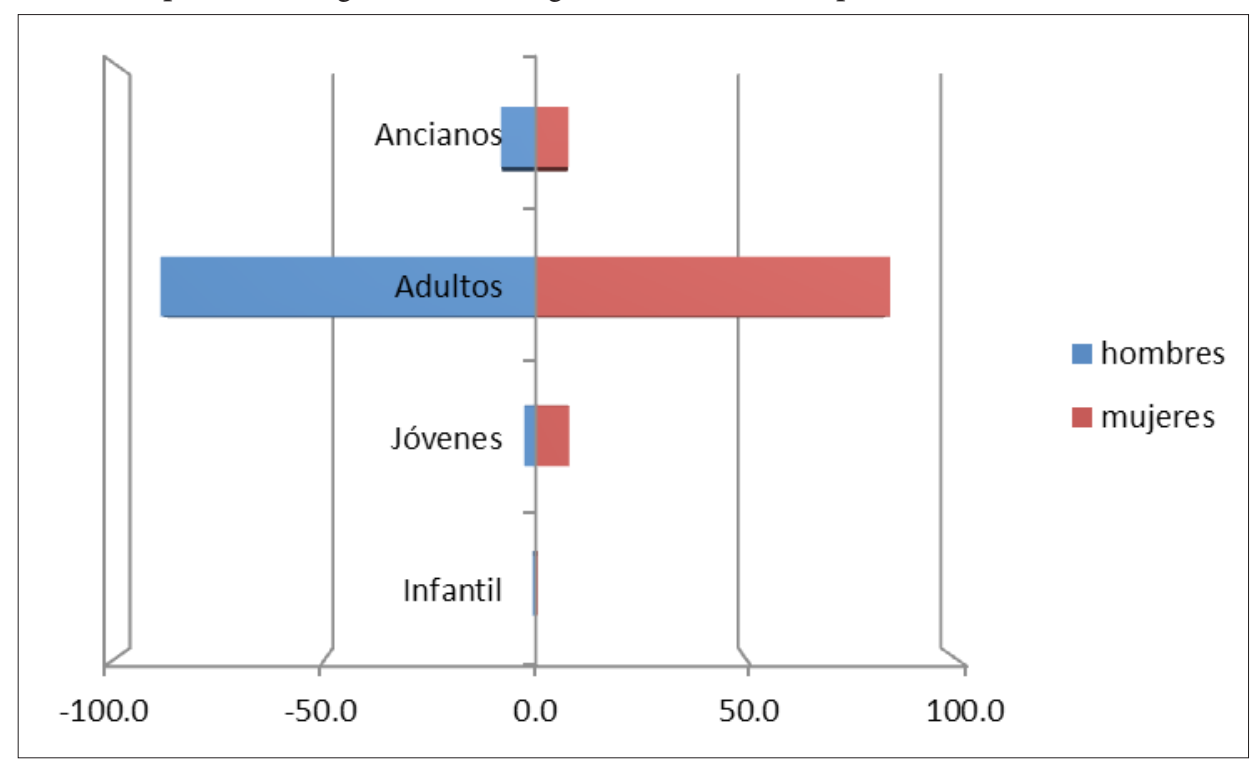

Fuente: Cálculos propios a partir de la ENSANUD, 2016.

En la figura 3 se aprecia la consecuencia más brutal que experimentan los diabéticos. En el 2016, del total de amputaciones de alguna parte de su cuerpo a causa de esta enfermedad, el $87 \%$ de los diabéticos del inframundo fueron amputados, respecto sólo al $13 \%$ de los diabéticos terrenales. Los últimos son los menos vulnerables a este daño porque son trabajadores formales. En cambio, los primeros, posiblemente llegan con una herida simple que no cicatriza y la primer acción emprendida en los hospitales es el corte de alguna parte de su cuerpo, entre ellas la más común es la del pié. 
Figura 3: México. Porcentaje de diabéticos amputados en alguna parte del cuerpo, según tipo de hospital en que se atendieron, 2016.

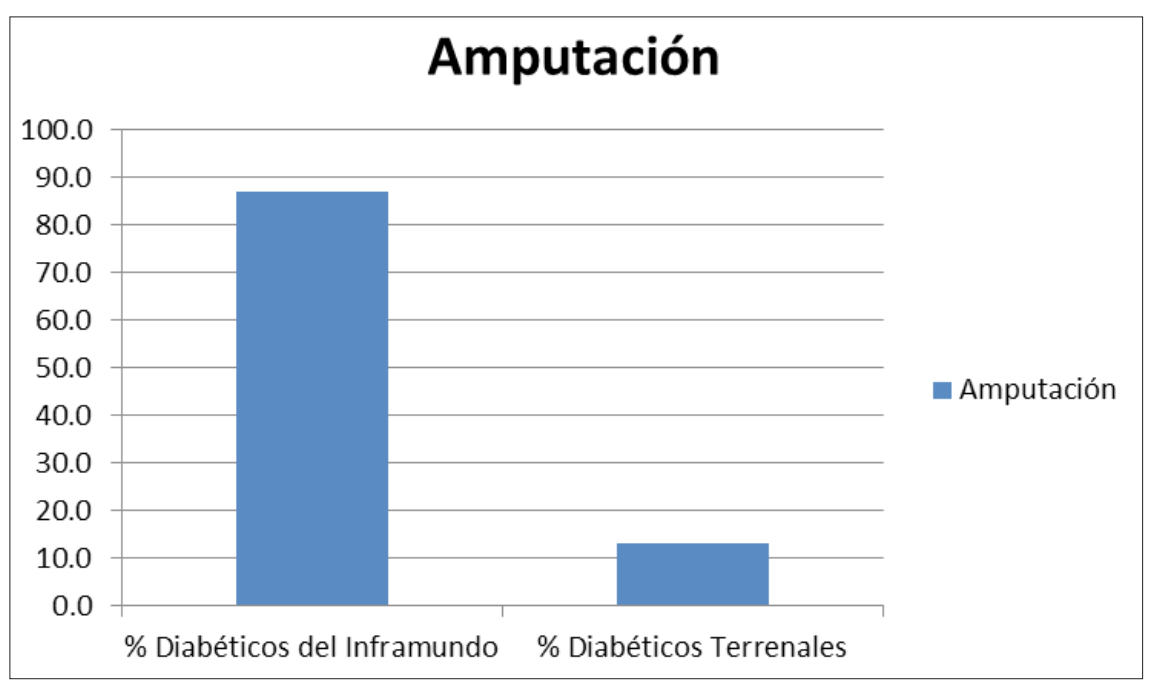

Fuente: Cálculos propios a partir de la ENSANUD, 2016.

El cuadro 3 desagrega el dato de amputación, según el hospital donde se atienden las personas para controlar su diabetes. Del total de amputaciones de los del inframundo, el $77.8 \%$ corresponden a los hospitales dependientes de farmacias. Aquí acudieron las personas y pagaron de su bolsillo por este tipo de agresión. El 8.0\% fueron amputados en hospitales de la Ssa, y el 1.1 en hospitales del Imss prospera. Los médicos privados, hospital civil, institutos nacionales y otros presentan niveles muy bajos de esta acción en la encuesta, por ese motivo no hay datos. Respecto a los terrenales, éstos son amputados en los hospitales públicos del Imss (8.4\%) y el Issste (4.7\%). Con base en que no hay registros de estas agresiones en los hospitales de los petroleros, de la marina y los militares, es posible que ahí tienen los recursos financieros para curar y salvar las extremidades de los enfermos (Cuadro 3).

Cuadro 3: México. Porcentaje de amputaciones según tipo de hospital donde se atendieron los diabéticos.

\begin{tabular}{|l|l|}
\hline Diabéticos del inframundo & \% de amputación \\
\hline Dependientes de farmacias & 77.8 \\
Ssa & 8.0 \\
Imss prospera & 1.1 \\
Médicos privados & 0.0 \\
Hospital civil & 0.0 \\
Institutos nacionales & 0.0 \\
Otro & 0.0 \\
\hline Diabéticos terrenales & $\mathbf{\%}$ de amputación \\
\hline Imss & 8.4 \\
Issste & 4.7 \\
Pemex & 0.0 \\
Defensa & 0.0 \\
Marina & 0.0 \\
Issste estatal & 0.0 \\
\hline Total & $\mathbf{1 0 0 . 0}$ \\
\hline
\end{tabular}

Fuente: Cálculos propios a partir de la ENSANUD, 2016. 


\section{DISCUSIÓN}

Lo expuesto hasta aquí deja claro que las enfermedades crónicas como la diabetes requieren un diseño participativo y específico de política pública que atienda el problema prioritario, principalmente para la población adulta. En este escenario, quedan muchas aristas por abordar por la falta de datos que evidencian las deficiencias en la cobertura sanitaria. Por ejemplo, partiendo de la población que asisitió a hospitales privados en el 2016 (el 26.4\% pagó por este servicio). La encuesta no registra los elevados montos con el que se capitalizan estos centros y con lo que han llegado a aglutinar la mayoría de quirófanos y de instrumental de alta tecnología, respecto a su contraparte pública.

En fín, la aportación del trabajo apunta a la confirmación de que la diabetes se concentra en edades laborales y que a los que más agreden con una amputación es a los que tienen empleo informal (diabéticos del inframundo) o que se ubican en la clase social baja. El dato es correcto, porque, recientemente se ha demostrado que en México, la enfermedad afecta más a la población con ingresos bajos (BARQUERA Y TOLENTINO, 2005).

En el país, la transición epidemiológica se ha acompañado del cambio de patrones de alimentación y actividad física. La carga de la enfermedad ya no se concentra en sectores sociales altos, pues la clase social baja es la más afectada por las enfermedades crónicas. La obesidad, como factor determinante se ha generalizado en todo el país. Si bien, en 1980 se concentraba en las entidades vecinas a los Estados Unidos, posiblemente, a causa de compartir los mismos patrones de alimentación; en el 2000, todas las regiones, incluyendo el centro, sur y la ciudad de México tenían niveles muy similares (BARQUERA Y TOLENTINO, 2005).

Por otro lado, los datos confirman los nexos existentes entre la diabetes y la edad laboral, en los que es probable que la ocurrencia de niveles altos de estrés, la edad y la posición socioeconómica sean las causas principales que están asociadas a dicha enfermedad. Al respecto, en Finlandia, con base en dos grandes muestras de adultos, tras confirmar el incremento en la probabilidad del riesgo de experimentar incapacidad permanente en el trabajo, pues se encontró asociación con el nivel de estrés laboral con las enfermedades crónicas como la diabetes (AIRAKSINEN, et al., 2017).

La gravedad de la epidemia de diabetes, así como el hecho de que se trata de una enfermedad prevenible, llama a fortalecer las estrategias para hacerle frente. El impacto que tiene sobre la calidad de vida de las personas que la padecen la convierte en un área prioritaria para el sector salud (HERNÁNDEZ, et al., 2013.

En Brasil se ha sugerido que la clave de la obesidad está el consumo de alimentos procesados como galletas, refresco, helados, etc., ya que ha disminuido el consumo de alimentos tradicionales como el frijol y el arroz (MONDINI, et al., 2007). Sin embargo, en las condiciones actuales del capitalismo, en las que se pone a la mano de la población trabajadora alimentos procesados, resulta sumamente difícil revertir este problema.

En México, en el mundo del trabajo, a pesar de que hay autores que afirman que el empleo ha llegado a su fin (RIFKIN, 2003; ANTUNES, 2005), el ideal del descanso es prácticamente inexistente. A excepción de las maquiladoras. No obstante estos negocios tienen condiciones de explotación de mano de obra barata (IGLESIAS, 1985). Mejor ellos ponen el ejemplo, ya que disponen de áreas de descanso, donde periódicamente realizan activación física, o tienen espacios para el consumo de alimentos. Mientras, en el resto de empleos, sean éstos públicos o privados, en instituciones de gobierno, escuelas, hospitales, organizaciones no gubernamentales. En todas ellas, se carecen de estas oportunidades.

Entonces, en el país, los trabajadores tienen que organizarse porque no es tan difícil imaginar en el corto plazo existan áreas de descanso en las escuelas por ejemplo, espacios donde se ofrezcan alimentos como frutas, verduras, agua, etc., además de lugares con música para hacer activación física, para disminuir el sedentarismo e intentar bajar el estrés laboral.

En países como Portugal, los comercios acostumbran una hora de siesta adicional a la hora de comida. En este tiempo, contrario a la lógica capitalista, las empresas ofrecen más tiempo de descanso para que sus empleados aguanten el resto de tiempo laboral.

Ricardo Antunes (2005) sostiene, la degradación del empleo se conduce a niveles nunca antes vistos. Primero, quienes están ocupados son exigidos cada 
vez más a hacer diversas actividades, cumplir con más responsabilidades y a capacitarse continuamente, todo a cambio de salarios muy bajos. Pero, el problema principal es para la población con desempleo estructural. Se trata del contingente creciente de trabajadores en condiciones tales de precarización que el mismo Marx definió como ejército industrial de reserva.

El estudio del respeto a los derechos humanos y la salud en México transita por cambios demográficos y epidemiológicos que necesitan la atención urgente, con el fin de buscar la mejora en la cobertura.

De acuerdo con Franco y Álvarez (2008), los derechos humanos son el marco apropiado para la acción en la salud pública. Así que con buena salud se sugiere terminarían problemas sociales como la pobreza, la marginación, la desigualdad, las diferencias de género y la violación de los derechos humanos.

En México, respecto a la cobertura, se puede asegurar que la población abierta, la socialmente más vulnerable no tiene garantizado el servicio sanitario en realidad, y es posible que sean ellos los que incrementan la probabilidad de muerte por esta causa.

\section{REFLEXIONES FINALES}

Este trabajo cumplió con el objetivo general y los objetivos específicos. Fundamentalmente, aporta reflexiones sugerentes sobre la importancia de la diabetes, útil para el análisis de la política pública de salud en México (2012-2016). Para este fin se ofrece evidencia del problema central de los servicios sanitarios, señal de alerta de que las cosas no funcionan bien. Contrario al diagnóstico de la secretaría de salud (2016). Ese mundo perfecto y maravilloso ha quedado deformado.

Siguiendo la literatura aquí revisada y con base en los resultados de los datos usados, en el marco de la política pública en salud se confirma la ocurrencia de problemas que atentan contra los derechos humanos. En este sentido, a los diabéticos mexicanos les urge el cambio en la atención de su salud.

De tal manera que, el principal reto que tienen los servicios sanitarios en los próximos años es cambiar la opción de la amputación que experimentan los diabéticos mexicanos. Porque, por donde se le vea está mal. El problema se reduce a dinero, ya que es más barato amputar, respecto del gasto oneroso que representa curar la parte afectada.

Respecto al primer objetivo específico se describe la proporción de la diabetes y la obesidad, según los derechos de cobertura en la población de 20 años, por sexo y grupos de edad.

El segundo objetivo de identificar la falta de respeto a los derechos humanos de los diabéticos, el trabajo ofrece la evidencia de la desproporción significativa de las amputaciones realizadas al tipo de diabético socialmente más vulnerable. En el 2016, del total, casi 9 de cada 10 de amputados son diabéticos del inframundo y sólo poco más de 1 de cada 10 corresponden a los diabéticos terrenales. Es fácil imaginar los atropellos con que se violenta el derecho a la salud de los primeros a causa del desconocimiento, determinado por el interés del dinero. Desafortunadamente, ellos son los que reciben el castigo más brutal de todos: la amputación de su cuerpo. Acción que parece ser la prioridad cuando los diabéticos ingresan a un hospital para recibir atención.

Entonces, el Estado tiene que intervenir porque en los hospitales dependientes de farmacias se han masificado las amputaciones en el 2016, luego destacan los hospitales públicos en el siguiente orden de importancia: Imss, Ssa e Issste.

El documento confirma también que las mujeres adultas son proporcionalmente más propensas a la enfermedad, respecto a los hombres. En este sentido, se necesitan nuevos estudios que exploren las asociaciones de la diabetes con el estrés laboral, el sedentarismo y la alimentación, sin olvidar el resto de variables como la genética, la edad, etc.

A su vez, en los próximos años, en el sistema de salud mexicano urgen datos sobre los cobros por la amputación y del costo del tratamiento del pié diabético. Por un lado, se tendría información en un ejercicio de transparencia que puede dar evidencia las estafas monetarias que ocurren a cambio de la salud. Por otra parte, transparentar los costos del tratamiento del pié diabético en hospitales de pemex, de la marina y los militares abonarían a ofrecer la misma oportunidad que no tiene el resto de la población.

Los problemas públicos necesariamente requiere la identificación correcta de los problemas públicos. La aportación aquí encontrada indica la necesidad urgente de substituir la opción de amputación, centralizar la política pública en salud 
en la población adulta y mejorar las condiciones de salud colectiva en los centros de trabajo.

Las posibilidades de estudio a futuro necesitan nuevos datos sobre la falta de respeto a los derechos humanos que van a la alza. Entre los temas a explorar son necesarios también documentar los abusos y negligencias médicas y la evolución de las condiciones laborales.

Las reflexiones centradas en el derecho y la salud permitieron apreciar que el peso de un mandato internacional parece corto comparado con las condiciones de la población. Es fácil ver que la decisión de la amputación tiene su origen en el desconocimiento popular, y que, el discurso del personal médico impera como verdadero y único. Aunque se trata de un abuso de poder. La pertinencia de los derechos humanos en salud y descanso necesita la participación de los trabajadores, de dotar de posibilidades para ser tomados en cuenta en el sistema de evaluación de los servicios de sanidad, para así también mejorar sus condiciones de vida.

Fermin Roland Schramm y Miguel Kottow (2001) sugieren que el fin de la evaluación de la política pública en salud debe servir para mejorar el servicio, con apego a la búsqueda del bien común y con base en el principio de protección.

La mejora en las condiciones de salud se alcanzarán por la vía del respeto de los derechos humanos. La salud pública, como bien público y la política pública tienen sentido si se apoyan en los derechos humanos mediante la construcción ciudadana y la participación política. De ahí que se tienen que promover.

Las limitaciones del estudio se centran en la decisión que tomé de elegir el tipo de diabético, dependiendo de la cobertura que tienen en los hospitales. Por ejemplo, el resultado aquí documentado referente a la amputación que ocurre mayoritariamente en un hospital privado, mismo que contiene, por supuesto, mayoritariamente a los diabéticos del inframundo y algunos de los terrenales, ya que todos ellos pagaron por este tipo de agresión.

Otra de las limitaciones del estudio viene de las deficiencias de cobertura que tienen los datos de las encuestas usadas, pues hay una subrepresentación de la población sin cobertura médica segura. En este sentido, es necesario además que la evaluación del estado de salud de los mexicanos la realice otra institución endógena, ajena a la secretaría de salud.

\section{REFERENCIAS}

AIRAKSINEN, J. Jokela, M. Virtanen, M. Oksanen, T. Pentti, J. Vahtera, J. Koskenvuo, M. Kawachi, I. Batty, G.D. y Kivimaki, M. Development and validation of the risk prediction model for work siability multicohort Study, Scientific Reports. 2017. Doi: 10.1038/s41598-017-13892-1.

AMERICAN DIABETES ASOCIATION. Obesity Management for the Treatment of Type 2 Diabetes, Diabetes Care Jan; 40, (1), U.S.A. 2017. https://doi.org/10.2337/ dc17-S010.

ANTUNES R. Los sentidos del trabajo. Ensayo sobre la confirmación y la negación del trabajo. Taller de estudios laborales, Ediciones Herramienta, Argentina, 2005. Consultado el 12 de septiembre de 2018 en:

https://es.scribd.com/doc/237352066/Ricardo-Antunes-Lossentidos-del-trabajo-pdf

BARQUERA, S. TOLENTINO, L. Geografía de las enfermedades asociadas con la nutrición en México: una perspectiva de transición epidemiológica. Instituto Nacional de Salud Pública en México, Papeles de Población, 11 (43), México, 2005, p. 134-139.

BASTERRA F.J. BES, M. RUIZ, M. GEA, A. MARTÍNEZ M.Á. Prevalencia de obesidad y diabetes en adultos españoles, 1987-2012. Medica Clínca, Barcelona, 2017, p. 250-256. https://doi.org/10.1016/j.medcli.2016.11.022

BOSCH X, ALFONSO F, BERMEJO J. Diabetes y enfermedad cardiovascular. Una mirada hacia la nueva epidemia del siglo XXI. Revista Especial Cardiol, 2002, p. 55:525.

CABRALES, O. La precarización laboral y el desempleo como consecuencias del neoliberalismo y la globalización; Revista Tendencias \& Retos, del programa de Trabajo Social de la Universidad De La Salle, México, 2011.

DANTÉS, O.G. SESMA, S. BECERRIL, V.M. KNAUL, F.M. ARREOLA, H. FRENK, J. Sistema de salud de México. Salud Pública, México, 2011, p. 320-232.

DIETERLEN, P. Justicia distributiva y pobreza, Coordenadas 2050, Universidad Autónoma de México, Cuadernos de la coordinación de Humanidades, México, 2016.

DURÁN, P. Transición epidemiológica nutricional o el "efecto mariposa", Archivo Argentino de Pediatría, 103 (3), Buenos Aires, 2005. Consultado el 18 de septiembre de 2018 en: http://www.scielo.org.ar/scielo.php?script=sci_arttext\& pid $=$ S0325-00752005000300002

ESTÉBANEZ, P. Los derechos humanos y el derecho a la salud. En: Estébanez P. Exclusión social y salud. Barcelona, Icaria, 2002. 
FERRANTE, D. LINETZKY, B. KONFINO, J. KING, A. VIRGOLINI, M. LASPIUR, S. Revista Argentina de Salud Pública, 2(6), Argentina, 2011, p. 34-41.

FRANCIA, Organización para la Cooperación y el Desarrollo Económicos. Estadísticas de la OCDE sobre la salud 2014, México, 2014. Consultado el 29 de agosto de 2018 en: www. oecd.org/health/healthdata

FRANCO, Á. ÁLVAREZ, C. Los derechos humanos, una oportunidad para las políticas públicas en salud. Gac Sanit, Barcelona, 22(3), 2008, p. 280-286.

FRENK, J. Elements for a theory of the Health Transition, México, 1991.

La salud de la población. Hacia una nueva salud pública, Fondo de cultura económica, México, 1995.

FRENK, J. LOZANO, R. BOBADILLA, J.L. La transición epidemiológica en América Latina. Revista Notas de Población; 22, México, 1994, p. 79-89.

GÓMEZ, O. SESMA, S. BECERRIL, V. KNAUL, F. ARREOLA, H. FRENK, J. Sistema de salud en México, Salud Pública, 53 (2), México, 2011.

GUATEMALA, Iniciativa Centroamericana de Diabetes (CAMDI) Encuesta de Diabetes, hipertensión y factores de riesgo de enfermedades crónicas. Villa Nueva, Guatemala, Organización Panamericana de la Salud, Guatemala, 2009. Consultado el 29 de agosto de 2018 en:

h t t p : / / i r s . pa ho.org/x m lui/bitstrea m/ handle/123456789/779/9275073996.pdf?sequence=1

HERNÁNDEZ, M. GUTIÉRREZ, J. REYNOSO, N. Diabetes mellitus en Mexico. Estado de la epidemia. Salud Pública, 55 (2), México, 2013. Consultado el 4 de septiembre de 2018 en:

https://www.scielosp.org/pdf/spm/2013.v55supp12/ s129-s136/es

HERON, M. Deaths: Leading Causes for 2016, National Vital Statistics Reports, 67 (6), U.S.A. 2018. Consultado el 4 de octubre de 2018 en:

file://C:/Users/Tomas/Downloads/cdc_57988_DS1.pdf

IGLESIAS, N. La flor más bella de la maquiladora, Centro de Estudios Fronterizos del Norte de México, Tijuana, México, 1985.

KLIL, A.J. AZOULAY, L. POLLAK, M.N. Cancer, obesity, diabetes, and antidiabetic drugs: is the fog clearing? Nature Reviews Clinical Oncology, vol. 14, 2016, p. 85-99.

LANDROVE, O. Reporte Técnico de Vigilancia, vol.10 (6), Cuba, 2005. Consultado en enero de 208 en: http://bvs.sld. $\mathrm{cu} /$ uats/rtv files/2005/landrove.htm

MANN, J. Voices from the past. Health and human rights: if not now, when? Am J Public Healt, 1996. Consultado el 10 de septiembre de 2018 en: http://www.ajph.org/cgi/content/extract/96/11/1940

MARRO, M.J., MOREYRA A., DA COSTA, L. Desigualdades regionales en la mortalidad por diabetes mellitus y en el acceso a la salud en Argentina, Cadernos Saúde Pública, 33 (9) Brazil, 2017.

MERINO, M. Políticas públicas. Ensayo sobre la intervención del Estado en la solución de problemas públicas, Primera reimpresión. CIDE, México, 2013.

MEXICO. Instituto Nacional de Geografía e Informática, Principales causas de mortalidad por residencia habitual, por grupos de edad y sexo del fallecido, México, 2018, Consultado el 4 de septiembre de 2018 en:

http:/www.inegi.org.mx/est/contenidos/proyectos/registros/ vitales/mortalidad/tabulados/ConsultaMortalidad.asp

MEXICO. Secretaría de salud. Informe sobre la salud de los mexicanos 2016. Diagnóstico general del sistema nacional de salud, México, 2016. Consultado el 20 de agosto de 2018 en: http:/www.gob.mx/salud/acciones-y-programas/direcciongeneral-de-evaluacion-del-desempeno?idiom=es-MX

MONDINI, L. LEVY, R.B. SALDIVA, S.R.D.M. VENÂNCIO, S.I. AGUILAR A.A. STEFANINI, M.L.R. Prevalência de sobrepeso e fatores associados em crianças ingressantes no ensino fundamental em um município da região metropolitana de São Paulo, Cade Saúde Pública, 23(8), Brazil, 2007. Consultado el 24 de agosto de 2018 en: https:/www.scielosp.org/pdf/csp/2007.v23n8/1825-1834/pt

NYBERG, S. et al. Job Strain as a Risk Factor for Type 2 Diabetes: A Pooled Analysis of 124,808 Men and Women, Diabetes Care, U.S.A, 2014. doi: 10.2337/dc13-2936

RIFKIN, J. El fin del trabajo. Nuevas tecnologías contra puestos de trabajo: el nacimiento de una nueva era. Revista Chilena de Derecho Informático, Chile, 2003. doi:10.5354/0717-9162.2011.10654.

REYNOSO, J. CARRILLO, J. ALGARÍN, L. CAMACHO, O. RUVALCABA, J.C. La obesidad y su asociación con otras de las enfermedades crónicas no transmisibles. Journal of Negative and No Positive Results: JONNPR, 3 (8), 2018, p. 627-642.

SCHRAMM, F.R. KOTTOW, M. Principios bioéticos en salud pública: limitaciones y propuestas. Cadernos de Saude Pública, 17, 2001; Consultado en: https://www.scielosp.org/ scielo.php?pid=S0102- 311 X2001000400029\&script $=$ sci arttext\&tlng=es

SHAW J. Diabetes, Síndrome metabólico y epidemia cardiovascular. Diabetes Voice, vol. 51, 2006.

U.S.A. Organización de las Naciones Unidas declaración universal de los derechos humanos, Nueva York, 1948. Consultado el 20 de agosto de 2018 en: https://www.ohchr. org/EN/UDHR/Documents/UDHR_Translations/spn.pdf 